\title{
CONHECIMENTO DE TUTORES DE CÃES SOBRE TUMOR DE MAMA EM CADELAS
}

\author{
[Knowledge of the dog's owners about mammary tumor in bitches]
}

\author{
Cláudia da Silva Magalhães ${ }^{1}$, Wagner Costa Lima², Dayanne Anunciação Silva Dantas Lima ${ }^{3}$, Ana Maria \\ Quessada $^{4^{*}}$, Daniel Enrico Müller Dornelles ${ }^{5}$, João Moreira da Costa Neto ${ }^{6}$ \\ ${ }^{1}$ Médica veterinária, doutoranda em Ciência Animal da Universidade Federal do Piauí (UFPI), docente do curso \\ de Medicina Veterinária da Universidade Federal de Roraima, Boa Vista, RR. \\ ${ }^{2}$ Médico veterinário docente do curso de Medicina Veterinária da UFPI, campus de Bom Jesus, Bom Jesus, PI. \\ 3Médica veterinária, Hospital Veterinário da UFPI de Bom Jesus, Bom Jesus, PI. \\ ${ }^{4}$ Médica veterinária, docente da pós-graduação em Ciência Animal da Universidade Paranaense (UNIPAR) e da \\ UFPI. \\ ${ }^{5}$ Acadêmico do curso de Medicina Veterinária da UFPI, campus de Teresina, Teresina, PI. \\ ${ }^{6}$ Médico-veterinário, Docente da Escola de Medicina Veterinária e Zootecnia, Universidade Federal da Bahia, \\ Salvador, BA
}

\begin{abstract}
RESUMO - Os tumores mamários (TM) são frequentes na espécie canina, sendo que a etiologia pode estar relacionada a diversos fatores, sendo o mais importante o fator hormonal. Devido a isso, a castração precoce é a principal forma de se prevenir a doença em cadelas. Este trabalho teve o objetivo de entrevistar tutores de cães na cidade de Teresina, a respeito do tumor de mama em cadelas. Foram abordadas 420 pessoas na cidade e observou-se que a maioria das pessoas não leva seu animal regularmente ao veterinário. Tal conduta diminui a possibilidade de diagnóstico precoce, fundamental em TM de cadelas. A maioria das pessoas respondeu que não castraria seu animal, demonstrando que não estão dispostos a adotar condutas importantes na guarda responsável. Dentre os que concordam com a castração, a anticoncepção foi apontada como principal motivo para muitos entrevistados. A maioria dos entrevistados $(71,19 \%)$ não sabia que cadelas podem ter TM e dentre as pessoas que afirmaram saber, a maioria não sabia como prevenir a doença. Apenas 8,24\% das pessoas citaram a castração antes do primeiro cio como conduta profilática para TM em cadelas. Diante dos resultados obtidos, constata-se que os habitantes de Teresina não possuem informações adequadas e suficientes a respeito de tumores mamários em cães. Sugere-se a instituição de campanhas educativas para diminuir a morbidade e mortalidade da enfermidade em cadelas.
\end{abstract}

Palavras-Chave: cães; informação; glândula mamária; profilaxia; proprietário.

\begin{abstract}
Mammary tumors (MT) are common in dogs, and the etiology may be related to several factors; however the most important factor is hormonal. Due to this, early castration is the main method to prevent the disease in dogs. This study aimed to interview dog guardians in the city of Teresina, about the mammary tumor in dogs. We addressed 420 people in the city and it was observed that most people do not take your pet to the veterinary regularly. Such behavior diminishes the possibility of early diagnosis, key in MT of female dogs. Most people do not accept to spay your animal, demonstrating that they are not willing to adopt important conduits in responsible ownership. Among those who agree with castration, contraception was identified as the main reason for many respondents. The most respondents $(71.19 \%)$ did not know that dogs may have MT and among the people who claimed to know, most did not know how to prevent the disease. Only $8.24 \%$ of people recognize spaying before the first heat as a prophylactic conduct for TM in dogs. Based on these results, it was concluded that the inhabitants of Teresina do not have adequate and sufficient information regarding mammary tumors in dogs. It is suggested the creation of educational campaigns to reduce the morbidity and mortality of the disease in dogs.
\end{abstract}

Keywords: dog; information; mammary gland; owner; prophylaxis.

\footnotetext{
* Autor para correspondência. E-mail: quessadavet@gmail.com Recebido: 28 de fevereiro de 2016.

Aceito para publicação: 17 de março de 2016.
} 


\section{INTRODUÇÃO}

Entre as diversas áreas existentes na clínica de cães, a oncologia veterinária tem se destacado na busca de melhorias na sanidade, prevenção, diagnóstico e tratamento de neoplasias (Nagata et al., 2014), já que tais enfermidades são afecções frequentes em cães. Em um estudo brasileiro, $62 \%$ dos diagnósticos de biópsias de animais (cães e gatos) referiam-se a neoplasias (Andrade et al., 2012).

Os tumores mamários (TM) são a mais frequente neoplasia em cães, sendo que em serviços de oncologia veterinária no Brasil, mais de $50 \%$ das neoplasias diagnosticadas em cães eram tumores mamários de cadelas (Ribas et al., 2012; Biondi et al., 2014). No Brasil, a maioria destes tumores é maligna, tendo sido detectada aproximadamente 90\% de malignidade em tumores mamários de cadelas (Toríbio et al., 2012; Biondi et al., 2014).

Apesar da importância epidemiológica de TM em cadelas no Brasil, os registros médicos de atendimento destes animais, são incompletos (Biondi et al., 2014), perdendo-se informações importantes principalmente na esfera reprodutiva como castração, ocorrência de enfermidades reprodutivas (pseudociese, aborto e outras) e uso de contraceptivos que são relacionadas à etiologia de TM em cadelas (Ribas et al., 2012; Toríbio et al., 2012).

A etiologia de TM pode estar relacionada a fatores de natureza dietética, genética, ambiental e hormonal (Ribas et al., 2012; Toríbio et al., 2012), com destaque para este último fator. Devido a isso, a castração precoce é reconhecida como a principal forma de se prevenir a doença em cadelas. Inclusive em fêmeas caninas já acometidas pela doença, mas com tumores benignos, a castração evita a formação de novos tumores em $50 \%$ das cadelas castradas após o diagnóstico (Kristiansen et al., 2013). Tais dados reforçam que a exposição hormonal durante a vida aumenta a predisposição para desenvolver TM e que a castração precoce (antes do primeiro cio) mostra bons resultados na redução do risco de desenvolver tais tumores (Kamiguchi et al., 2016).

Diante do exposto, este trabalho teve o objetivo de entrevistar tutores de cães na cidade deTeresina, a respeito do tumor de mama em cadelas. $\mathrm{O}$ trabalho foi aprovado pelo comitê de ética humano da Universidade Federal do Piauí sob protocolo 094/2010.

\section{MATERIAL E MÉTODOS}

Foram entrevistadas 420 pessoas (tutoras de cães ou não) que foram abordadas por meio de seleção aleatória em diferentes pontos da cidade de Teresina.

Para o cálculo amostral dos habitantes de Teresina, foi levada em consideração a população do município que, segundo o censo do IBGE (2010) possui 814.230 habitantes. Foi utilizada a seguinte fórmula (Marotti et al., 2008):

$$
\mathrm{N}=\frac{\partial^{2} \cdot p \cdot q \cdot N}{\mathrm{e}^{2} \cdot(\mathrm{N}-1)+\partial^{2} \cdot p \cdot q}
$$

$\mathrm{N}$ : População de Teresina; $\partial$ : equivalência do erro (1,96 2); p: proporção de indivíduos na categoria estudada, q: proporção de indivíduos não pertencente à categoria estudada; e: erro (estimado em 5\%; intervalo de confiança, $95 \%$ ).

$$
\begin{gathered}
\mathrm{N}=\frac{(2)^{2} \cdot 50.50814 .230}{(5)^{2} .}(814.230-1)+2^{2} .50 \cdot 50 \\
\mathrm{~N}=399,80
\end{gathered}
$$

Portanto, levando-se em consideração tal cálculo, seriam entrevistadas 400 pessoas, para maior segurança 420 habitantes foram incluídos no estudo. Foram incluídas nas entrevistas perguntas sobre animais e sobre TM em cadelas (principalmente etiologia e prevenção) e todos os dados obtidos foram analisados por métodos percentuais.

\section{RESULTADOS E DISCUSSÃO}

Entre os entrevistados (420 pessoas), verificou-se que $75,47 \%$ (317/420) eram tutores de animais, tendo a maioria destes $(98,57 \%$; 414/420) cães sob sua responsabilidade, demonstrando que os cães são os preferidos como animais de estimação no Brasil, o que pode ser comprovado pela presença constante dos cães na mídia brasileira (Würdig, 2014). Além disso, alguns estudos registraram que os cães são os animais preferidos para companhia no Brasil (Catapan et al., 2015; Toscano et al., 2015) Essa preferência pode ser explicada devido à confiança que os cães despertam em seus proprietários, além de proporcionarem afeto, contato corporal e proteção (Catapan et al., 2015).

A maioria dos entrevistados $(56,19 \% ; 236 / 420)$ não leva seu animal regularmente ao veterinário e o leva somente quando já doente $(53,57 \% ; 225 / 420)$. Tal resultado difere do encontrado em um estudo paranaense onde a maioria das pessoas procura assistência veterinária regularmente (Catapan et al., 2015). Provavelmente tal diferença está relacionada a diferenças socioeconômicas entre as regiões abordadas. A conduta detectada na pesquisa em questão diminui a possibilidade de diagnóstico 
precoce, fundamental em TM de cadelas (Gómez, Ramírez, Maldonado, 2012; Petrov et al., 2014).

A maioria das pessoas $(63,09 \%$; 225/420) respondeu que não castraria seu animal, demonstrando que não estão dispostos a adotar condutas importantes na guarda responsável como a castração (Catapan et al., 2015), que tem a capacidade de diminuir a taxa de natalidade de cães e gatos em uma localidade (Catapan et al., 2014). Em pesquisa realizada no Paraná, conduta semelhante à registrada neste estudo foi observada. No estudo citado, a maioria das pessoas é favorável à castração de animais de rua, mas a maioria não castrou seu próprio animal (Catapan et al., 2015), demostrando que os gestores brasileiros precisam adotar medidas educativas em todos os setores da sociedade.

Dentre os que concordam com a castração $(36,90 \%$; 155/420), a anticoncepção foi apontada como principal motivo para muitos entrevistados (40,00\%; 62/155), conforme detectado em outro estudo (Toscano et al., 2015).

Muitos artigos brasileiros que foram consultados para embasar esta pesquisa demonstram que a maioria das cadelas acometidas por tumor de mama não são castradas (Feliciano et al., 2012; Ribas et al., 2012; Toríbio et al., 2012; Biondi et al., 2014; Kamiguchi et al., 2016), o que pode denotar desconhecimento dos tutores sobre a proteção proporcionada pela castração precoce das fêmeas. Inclusive em um programa de esterilização cirúrgica de cães e gatos observou-se que a média de idade das cadelas castradas foi de 2,9 anos, demonstrando que a maioria foi de fêmeas adultas (Catapan et al., 2014). Corroborando estes fatos, observou-se que a maioria dos entrevistados $(71,19 \%$; 299/420) (Figura 1) não sabia que cadelas podem ter TM e dentre as pessoas que afirmaram saber $(28,81 \%$; 121/420) (Figura 1$)$, a maioria não sabia como prevenir a doença $(73,88 \% ; 89 / 121)$. Apenas 8,24\%; (10/121) citaram a castração antes do primeiro cio como conduta profilática para TM em cadelas. Desta maneira, as neoplasias mamárias em cadelas são frequentes no Brasil (Ribas et al., 2012; Toríbio et al., 2012), o que leva à afirmação de que é urgente educar a população quanto à prevenção e detecção da neoplasia mamária nas cadelas, buscando o diagnóstico precoce e a terapêutica adequada (Toríbio et al., 2012).

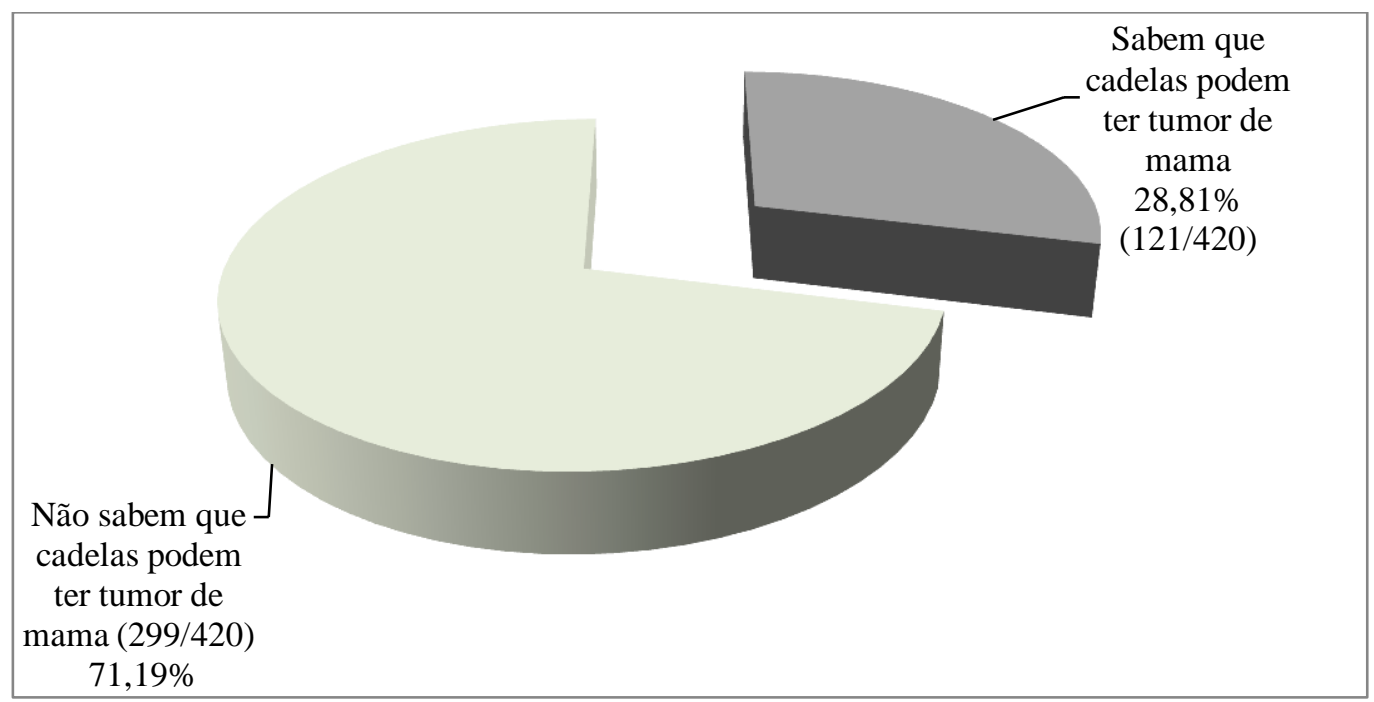

Figura 1. Conhecimento de pessoas entrevistadas em Teresina (PI) sobre tumor de mama em cadelas $(n=420)$.

A castração é medida profilática fundamental em tumor de mama em cadelas devido à etiologia hormonal e bastante importante na epidemiologia. No entanto, outros fatores também são importantes, especialmente o escore corporal. Alguns trabalhos demonstram que a obesidade está relacionada ao aparecimento de tumor de mama em cadelas (Ribas et al., 2012; Toríbio et al., 2012). Porém nenhum entrevistado citou a importância de manutenção do peso ideal como profilaxia de tumor de mama na espécie. Tal achado reforça ainda mais a ideia de que há necessidade de fornecer informação aos proprietários sobre prevenção, diagnóstico precoce e tratamento das neoplasias mamárias, principalmente nos locais onde há menor índice de instrução e renda (Toríbio et al., 2012), como é o caso de Teresina.

A diminuição dos casos de tumor de mama em cadelas pode também diminuir os casos de 
abandono de animais. Em muitos centros de zoonoses no Brasil uma das principais causas de abandono de cães são as enfermidades (Almeida et al., 2014), entre elas os tumores de mama em cadelas.

\section{CONCLUSÃO}

Diante dos resultados obtidos, constata-se que os habitantes de Teresina não possuem informações adequadas e suficientes a respeito de tumores mamários em cães. A maioria dos tutores demonstrou desconhecimento da existência, causa e profilaxia da enfermidade. Sugere-se que médicos veterinários, instituições de ensino de medicina veterinária, órgãos de classe, sociedades protetoras e gestores públicos invistam em campanhas educativas de posse responsável para diminuir a morbidade e mortalidade da enfermidade em cadelas.

\section{REFERÊNCIAS}

ALMEIDA, E.S. et al. Estudo do destino dos cães no canil municipal de Botucatu antes e após a lei $n^{\circ} 12.916$ que dispõe sobre o controle da reprodução de cães. Veterinária e Zootecnia, v.21, n.3, p.433-439, 2014.

ANDRADE, R.L.F.S. et al. Tumores de cães e gatos diagnosticados no semiárido da Paraíba. Pesquisa Veterinária Brasileira, v. 32, n.10, p.1037-1040, 2012.

BIONDI, L.R. et al. Canine mammary tumors in Santos, Brazil: clinicopathological and survival profile. Brazilian Journal of Veterinary Research and Animal Science, v. 51, n. 3, p. 252262, 2014.

CATAPAN, D.C. et al. Impacto do programa de esterilização cirúrgica na população de cães e gatos do município de São José dos Pinhais - PR. Revista brasileira de Ciência Veterinária, v.21, n.3, p.179-182, 2014.

CATAPAN, D.C. et al. Percepção e atitudes do ser humano sobre guarda responsável, zoonoses, controle populacional e cães em vias públicas. Revista brasileira de Ciência Veterinária, v. 22, n. 2, p. 92-98, 2015.

FELICIANO, M.A.R. et al. Estudo clínico, histopatológico e imunoistoquímico de neoplasias mamárias em cadelas. Arquivo Brasileiro de Medicina Veterinária e Zootecnia, v.64, n.5, p.1094-1100, 2012

GÓMEZ, B.; RAMÍREZ, M.; MALDONADO, J. Presence of lung metastases in bitches affected by malignant mammary neoplasms in Medellin (Colombia). Revista MVZ, v. 17, n.2, p.2983-2990, 2012.

IBGE. Censo demográfico 2010. Disponível em: http://www.cidades.ibge.gov.br/xtras/home.php.

KAMIGUCHI, I.E. et al. Mammary Neoplasms in Female Dogs: Identification of Cytopathological Criteria for Malignancy. Journal of Cytology and Histology, v.7, n.1, p.

KRISTIANSEN, V.M. et al. Effect of Ovariohysterectomy at the Time of Tumor Removal in Dogs with Benign Mammary Tumors and Hyperplastic Lesions: A Randomized Controlled Clinical Trial. Journal of Veterinary Internal Medicine, v. 27, n. 4, p. 935-942, 2013.
MAROTTI, J. et al. Amostragem em Pesquisa Clínica: tamanho da amostra. Revista de Odontologia da Universidade Cidade de São Paulo, v. 20, n.2, p. 186-194, 2008.

NAGATA, W.B. Perfil epidemiológico da neoplasia mamária canina em Araçatuba: uma abordagem estatística. Revista de Estatística da UFOP, v.3, n.3, p. 669-673, 2014.

PETROV, E.A. et al. Canine mammary tumours - clinical survey. Macedonian Veterinary Review, v. 37, n.2, p.129-134, 2014 .

RIBAS, C.R. et al. Alterações clínicas relevantes em cadelas com neoplasias mamárias estadiadas. Archives of Veterinary Science, v.17, n.1, p.60-68, 2012.

TORÍBIO, J.M.M.L. et al. Caracterização clínica, diagnóstico histopatológico e distribuição geográfica das neoplasias mamárias em cadelas de Salvador, Bahia. Revista Ceres, v. 59, n. 4 , p. 427-433, 2012.

TOSCANO, J.H.B. et al. percepção dos proprietários de animais de companhia sobre guarda responsável no município de Jaboticabal- SP. Ars Veterinaria, v.31, n.2, p.88, 2015.

WÜRDIG, R.C. As crianças, os animais e suas brincadeiras: um traço importante da cultura lúdica. Revist Aleph, v.11, n.22, p. 265-279, 2014. 